\title{
Pathalogical Lab Image Retrieval as an Alternative Expert
}

\author{
Sonali Pawar \\ Vidyalankar Institute of \\ Technology \\ Vidyalankar college marg, \\ Wadala. Mumbai-400037
}

\author{
Shivika Pandita \\ Vidyalankar Institute of \\ Technology \\ Vidyalankar college marg, \\ Wadala. Mumbai-400037
}

\author{
Mrunmayee Salunke \\ Vidyalankar Institute of \\ Technology \\ Vidyalankar college marg, \\ Wadala. Mumbai-400037
}

\begin{abstract}
Image retrieval in the real world is rapidly growing which has created innumerable possibilities for real world image search system. In this project inputs will be taken in the form of text and images. Based on that, disease will be detected and detailed report will be generated in case the doctor is not available. This project will be of great help in Pathology laboratories located in Rural areas where MD doctors are rare to be found. This system would be an alternative expert which will prove to be time saving. The text input of the system can we used by medical students for learning. Also the report generated by the system can be linked to social security number and used in case of emergencies as well as solving criminal cases and numerous other purposes.
\end{abstract}

\section{General Terms}

SURF - Speeded up robust feature, Image retrieval, Dictionary based algorithm, Feature extraction based on color, size and shape, preprocessing using various filters.

\section{Keywords}

Image retrieval; disease detection; color; shape; size; report generation.

\section{INTRODUCTION}

Earlier time it had to be counted under microscope which was time consuming \&amp; error prone but today there are many automated counters which are fast \&amp; can reduce error up to high percentage. In this proposed project with the help of scanned images and texts as a query we can identify the problems and provide valuable information to pathologist and for diagnosis and treatment of many diseases. Feature (content) extraction is the basis of content-based image retrieval. In a broad sense, features may include both textbased features (key words, annotations) and visual features (color, texture, shape). However, since there already exists rich literature on text-based feature extraction in the DBMS and information retrieval research communities, we will confine ourselves to the techniques of visual feature extraction. The technology used for this project will be Matlab, since MATLAB is considered to be the best platform to implement image processing algorithms. Also the system will consist of GUI which will also be developed using MATLAB UI tools. With the help of this project we provide preliminary treatment to the patients even if the doctor is unavailable. It is also a time saving and cost efficient project Content-based image retrieval (CBIR) has been one on the most vivid research areas in the field of computer vision over the last 10 years. The availability of large and steadily growing amounts of visual and multimedia data, and the development of the Internet underline the need to create thematic access methods that offer more than simple text- based queries or requests based on matching exact database field. Many programs and tools have been developed to formulate and execute queries based on the visual or audio content and to help browsing large multimedia repositories. Still, no general breakthrough has been achieved with respect to large varied databases with documents of differing sorts and with varying characteristics. Answers to many questions with respect to speed, semantic descriptors or objective image interpretations are still unanswered. This project gives an overview of available literature in the field of content-based access to medical image data and on the technologies used in the field. A demand for easy retrieval of images matching a query condition so that this database can be used as a clinical decision support system. This proposed project presents an efficient technique for retrieval of disease positive images from a clinical image database, that match a query condition mentioned in text or as another disease positive image.

\section{LITERATURE SURVEY}

1. Anik K.jain and Aditya Vailaya in their paper "Image retrieval using color and shape" emphasize on the fact that retrieval accuracy on the basis of color is better than that based on shape. Integrating the results of the color- and shape-based queries provides a better and more robust performance than either of the individual feature-based queries. Humans also use a combination of features (color, shape, and texture) to recognize objects and do not rely on any one individual feature. The speed of retrievals can also be increased by using a branch and bound method to compute the nearest neighbors for the query image without affecting the robustness of the system.[3]

2. Rui ,Yong, Thomas S. Huang and Shih-Fu Chang in their paper "Current techniques, promising directions, and open issues" provides comprehensive survey of the technical achievements in the research area of image retrieval, especially content-based image retrieval. It covers the research aspects of image feature representation and extraction, multidimensional indexing, and system design, three of the fundamental bases of contentbased image retrieval. For each visual feature, there exist multiple representations which model the human perception of that feature from different perspectives. What features and representations should be used in image retrieval is application dependent. There is a need of developing an image content description (model) to organize the features. The features should not only be just associated with the images, but also they 46 RUI, HUANG, AND 
CHANG should be invoked at the right place and the right time, whenever they are needed to assist Retrieval.[4]

3. Henning Muller, Nicholas Michoux and David Bandon in their paper "A review of content- based image retrieval systems in medical applicationsclinical benefits and future directions" talk about Content-based visual information retrieval (CBVIR) or content-based image retrieval (CBIR). In their view it has been one of the most vivid research areas in the field of computer vision over the last 10 years. This article gives an overview of available literature in the field of content-based access to medical image data and on the technologies used in the field. Section 1 gives an introduction into generic content-based image retrieval and the technologies used. Section 2 explains the propositions for the use of image retrieval in medical practice and the various approaches. Example systems and application areas are described. Section 3 describes the techniques used in the implemented systems, their datasets and evaluations. Section 4 identifies possible clinical benefits of image retrieval systems in clinical practice as well as in research and education. New research directions are being defined that can prove to be useful. They also state that that the goal is not, in general, to replace text-based retrieval methods as they exist at the moment but to complement them with visual search tool.[7]

4. Gudivada, Venkat N., and Vijay V. Raghavan.in their paper "Content based image retrieval system" has explained that images are being generated at an ever-increasing rate by sources such as defence and civilian satellites, military reconnaissance and surveillance flights, fingerprinting and mug-shotcapturing devices, scientific experiments, biomedical imaging, and home entertainment systems. A content-based image retrieval (CBIR) system is required to effectively and efficiently use information from these image repositories. Such a system helps users (even those unfamiliar with the database) retrieve relevant images based on their contents. Application areas in which CBIR is a principal activity are numerous and diverse. With the recent interest in multimedia systems, CBIR has attracted the attention of researchers across several disciplines.it involves the idea of the image search in large databases. [5]

\section{ALGORITHM}

Speeded Up Robust Features (SURF ) In computer vision, speeded up robust features (SURF) is a patented local feature detector and descriptor. It can be used for tasks such as object recognition, image registration, classification or 3D reconstruction. It is partly inspired by the scale-invariant feature transform (SIFT) descriptor. The standard version of SURF is several times faster than SIFT and claimed by its authors to be more robust against different image transformations than SIFT SURF descriptors have been used to locate and recognize objects, people or faces, to reconstruct 3D scenes, to track objects and to extract points of interest. SURF was first presented by Herbert Bay, et al., at the 2006 European Conference on Computer Vision. An application of the algorithm is patented in the United States version of SURF (called U-SURF) is not invariant to image rotation and therefore faster to compute and better suited for application where the camera remains more or less horizontal.

\section{Feature Detection}

1] Edge Detection

\section{a. Canny Edge Detection}

The Canny edge detector is an edge detection operator that uses a multi-stage algorithm to detect a wide range of edges in images. It was developed by John F. Canny in 1986. Canny also produced a computational theory of edge detection explaining why the technique works.Process of Canny edge detection algorithm The Process of Canny edge detection algorithm can be broken down to 5 different

1. Apply Gaussian filter to smooth the image in order to remove the noise

\section{Find the intensity gradients of the image}

3. Apply non-maximum suppression to get rid of spurious response to edge detection

\section{Apply double threshold to determine potential edges}

5. Track edge by hysteresis: Finalize the detection of edges by suppressing all the other edges that are weak and not connected to strong edges.

\section{2] Hough Transform}

The Hough transform is a feature extraction technique used in image analysis, computer vision, and digital image processing. [1] The purpose of the technique is to find imperfect instances of objects within a certain class of shapes by a voting procedure. This voting procedure is carried out in a parameter space, from which object candidates are obtained as local maxima in a so-called accumulator space that is explicitly constructed by the algorithm for computing the Hough transform.

a. In automated analysis of digital images, a subproblem often arises of detecting simple shapes, such as straight lines, circles or ellipses. In many cases an edge detector can be used as a pre-processing stage to obtain image points or image pixels that are on the desired curve in the image space. Due to imperfections in either the image data or the edge detector, however, there may be missing points or pixels on the desired curves as well as spatial deviations between the ideal line/circle/ellipse and the noisy edge points as they are obtained from the edge detector. For these reasons, it is often non-trivial to group the extracted edge features to an appropriate set of lines, circles or ellipses. The purpose of the Hough transform is to address this problem by making it possible to perform groupings of edge points into object candidates by performing an explicit voting procedure oer a set of parameterized image objects (Shapiro and Stockman, 304).

b. The simplest case of Hough transform is detecting straight lines. In general, the straight line $\mathrm{y}=\mathrm{mx}+\mathrm{b}$ can be represented as a point $(b, m)$ in the parameter space. However, vertical lines pose a problem. They would give rise to unbounded values of the slope parameter $\mathrm{m}$. Thus, for computational reasons, Duda and Hart proposed the use of the Hesse normal form

$\mathrm{R}=\mathrm{x} \operatorname{Cos} \Theta+\mathrm{y} \operatorname{Sin} \Theta$

where $\mathrm{R}$ is the distance from the origin to the closest point on the straight line and (theta) is the angle between the axis and 
the line connecting the origin with that closest point. It is therefore possible to associate with each line of the image a pair .The plane is sometimes referred to as Hough space for the set of straight lines in two dimensions. This representation makes the Hough transform conceptually very close to the two-dimensional Radon transform. (They can be seen as different ways of looking at the same transform. Given a single point in the plane, then the set of all straight lines going through that point corresponds to a sinusoidal curve in the $(\mathrm{r}, \theta)$ plane, which is unique to that point. A set of two or more points that form a straight line will produce sinusoids which cross at the $(r, \theta)$ for that line. Thus, the problem of detecting collinear points can be converted to the problem of finding concurrent curves.

\section{Affine invariant feature detection}

a. Harris affine region detector

In the fields of computer vision and image analysis, the Harris affine region detector belongs to the category of feature detection. Feature detection is a preprocessing step of several algorithms that rely on identifying characteristic points or interest points so to make correspondences between images, recognize textures, categorize objects or build panoramas. The Harris affine detector can identify similar regions between images that are related through affine transformations and have different illuminations. These affine-invariant detectors should be capable of identifying similar regions in images taken from different viewpoints that are related by a simple geometric transformation: scaling, rotation and shearing. These detected regions have been called both invariant and covariant. On one hand, the regions are detected invariant of the image transformation but the regions covariantly change with transformation. Do not dwell too much on these two naming conventions; the important thing to understand is that the design of these interest points will make them compatible across images taken from several viewpoints. Other detectors that are affine-invariant include Hessian affine region detector, Maximally stable extremal regions, Kadir-Brady saliency detector, edge-based regions (EBR) and intensityextrema- based regions (IBR).

\section{FLOWCHART}

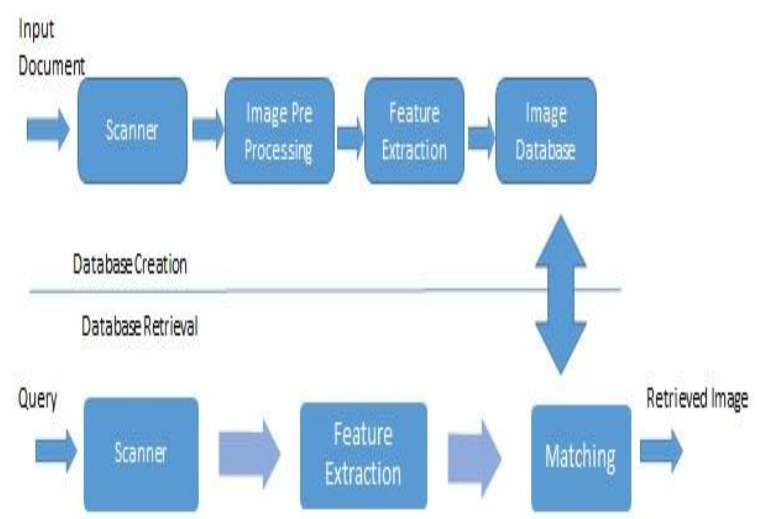

Fig 1. Methodology of Image Retrieval
Above Fig 1 shows a block diagram of the proposed image retrieval scheme. The input images are preprocessed to extract the features which are then stored along with the images in the database. When a query image is presented, it is similarly preprocessed to extract its features which are then matched with the feature vectors present in the database. A ranked set of images with high matching scores are presented at the output

\section{ACKNOWLEDGMENTS}

We have taken efforts in this project. However, it would not have been possible without the kind support and help of many individuals and organizations. I would like to extend my sincere thanks to all of them. We are highly indebted to Prof. Bhanu Tekwani for her guidance and constant supervision as well as for providing necessary information regarding the project \&amp; also for her support in completing the project. We would like to express gratitude towards our parents and; member of Vidyalankar Institute of Technology for their kind co-operation and encouragement which helped us in completion of this project. Our thanks and appreciations also goes to our colleagues in developing the project and people who have willingly helped us out with their abilities.

\section{REFERENCES}

[1] Rui, Yong, et al. "Relevance feedback: a power tool for interactive content-based image retrieval."IEEE Transactions on circuits and systems for video technology 8.5 (1998): 644-655.

[2] Rui, Yong, Thomas S. Huang, and Shih-Fu Chang. "Image retrieval: Current techniques, promising directions, and open issues." Journal of visual communication and image representation 10.1 (1999): 39-62.

[3] Jain, Anil K., and Aditya Vailaya. "Image retrieval using color and shape." Pattern recognition 29.8 (1996): 1233-1244.

[4] Rui, Yong, et al. "Relevance feedback: a power tool for interactive content-based image retrieval."IEEE Transactions on circuits and systems for video technology 8.5 (1998): 644-655.

[5] Gudivada, Venkat N., and Vijay V. Raghavan. "Content based image retrieval systems."Computer 28.9 (1995): $18-22$.

[6] Datta, Ritendra, et al. "Image retrieval: Ideas, influences, and trends of the new age." ACM Computing Surveys (CSUR) 40.2 (2008)

[7] Henning Muller, Nicholas Michoux and David Bandon. "A review of content-based image retrieval systems in medical applications - clinical benefits and future directions". Service of Medical Informatics, University Hospital of Geneva, Rue Micheli-du-Crest 24, 1211 Geneva 14, Switzerland 